\title{
Article \\ Optical Filter with Large Angular Dependence of Transmittance Using Liquid Crystal Devices
}

\author{
Kohki Takatoh *(1), Masahiro Ito $\mathbb{B}$, Suguru Saito and Yuuta Takagi \\ Department of Electrical Engineering, Faculty of Engineering, Sanyo-Onoda City University, 1-1-1 Daigaku-dori, \\ Sanyo-Onoda, Yamaguchi 756-0884, Japan; m-ito@rs.socu.ac.jp (M.I.); F216025@ed.socu.ac.jp (S.S.); \\ F215057@ed.socu.ac.jp (Y.T.) \\ * Correspondence: takatoh@rs.socu.ac.jp; Tel.:+81-836-88-4544
}

check for updates

Citation: Takatoh, K.; Ito, M.; Saito, S.; Takagi, Y. Optical Filter with Large Angular Dependence of Transmittance Using Liquid Crystal Devices. Crystals 2021, 11, 1199. https://doi.org/10.3390/cryst11101199

Academic Editor: Anatoliy

V. Glushchenko

Received: 7 September 2021

Accepted: 30 September 2021

Published: 3 October 2021

Publisher's Note: MDPI stays neutral with regard to jurisdictional claims in published maps and institutional affiliations.

Copyright: (c) 2021 by the authors. Licensee MDPI, Basel, Switzerland. This article is an open access article distributed under the terms and conditions of the Creative Commons Attribution (CC BY) license (https:// creativecommons.org/licenses/by/ $4.0 /)$.
Abstract: This study proposed a new type of optical device with variable transmittance based on the incident angle direction. These devices consist of two liquid crystal devices (LCDs) with a half-wave plate between them. Hybrid aligned nematic (HAN)-type guest-host (GH) LCDs or GH-LCDs with antiparallel alignment of high pretilt angles were used. The use of a half-wave plate allowed for the control of the $\mathrm{p}$ - and s-waves. Using these devices, a wide range of transmittances were obtained because no polarizer was used. The newly proposed LCDs have a wide range of applications, including use on buildings, vehicles, and glasses.

Keywords: liquid crystal; incident angle dependence; transmittance; HAN-LCD; hybrid aligned nematic; high pretilt LCD; half-wave plate; smart window; sunglasses; vehicle window

\section{Introduction}

Light control is extremely important for observing objects correctly without stress. In particular, the light observed directly from the sky to the eyes can be a large hindrance to observing an object correctly. Under typical conditions, objects are observed in the front or lower direction. Therefore, optical filters whose transmittance is small in the upward direction and large in the front or downward directions should be valuable.

Figure 1 shows the function of the optical filter with the incident angular dependence of the transmittance, which presents as a "louver" function. The louver can reduce the light only from the upward direction. Thin optical films with this function have a variety of applications, such as buildings, vehicles, and glasses. It can also be valuable if the transmittance is controlled by the electric field. This investigation used guest-host (GH) liquid crystal (LC) devices for these applications. GH-liquid crystal devices (LCDs) are devices that use dichroic dyes without polarizers. The dye molecules are aligned parallel to the molecular axis of the LC and absorb polarized light vibrating parallel to the molecular axis.

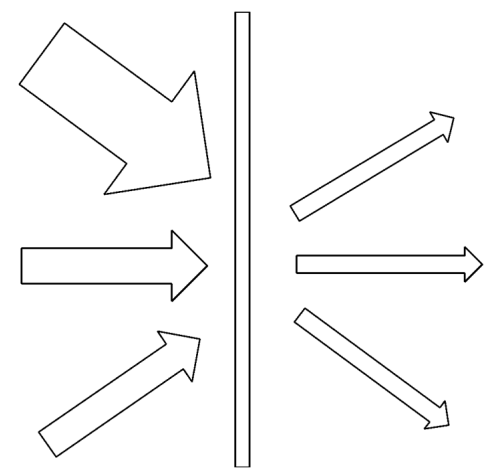

Figure 1. Optical filter of which transmittance can be controlled depending on the incident angles. 
By using the structure in which the dye molecules decline to the substrate plane, the incident light propagating along the dye molecules passes through the molecules with little absorption, and the light moving perpendicular to the molecular axis is absorbed more effectively. Two types of LCDs possess this structure. The first is the hybrid aligned nematic (HAN) and the other is the high pretilt angle LCDs. High pretilt angle LCDs are defined by alignment layers showing a high pretilt angle, and the directions of LC molecular declination on both substrates are opposite. The structures of these types of LCDs are shown in Figure 2a,b.

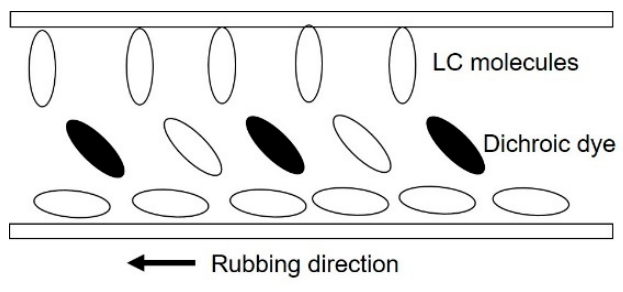

(a)

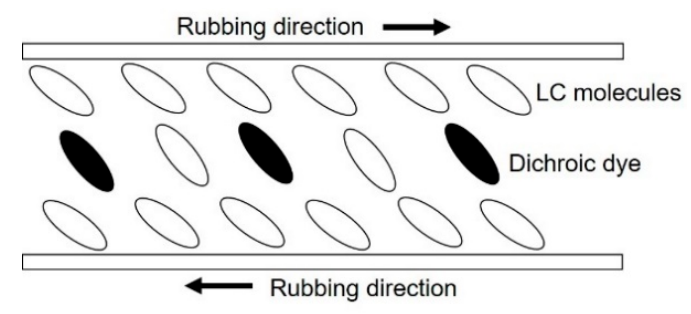

(b)

Figure 2. (a) Structure of HAN GH-LCDs; (b) Structure of high pretilt angle GH-LCDs.

As shown in Figure 2a, the two substrates of HAN GH-LCDs possess two types of alignment layers, namely a homogeneous and a homeotropic layer. The polar angle of LC molecules varies from 2 or 3 degrees (the pretilt angle of the homogeneous alignment layer) to $90^{\circ}$ continuously from the surface of the homogeneous alignment layer to that of the homeotropic alignment layer. Therefore, the molecules at the center of the LC layer are inclined at approximately $45^{\circ}$ from the substrate surface plane.

It was proposed that by using an inclined molecular arrangement, the incident angle dependence of the transmittance can be controlled. The reverse polymer-dispersed LC (PD-LC) mode with a HAN structure, which causes light scattering when an electric field is applied, shows the incident angle dependence of the transmittance [1-3]. This application has been proposed for smart windows as well as displays and other applications [4-7]. In these papers, except for the usage of the light scattering phenomenon [1-3], polarized light was used. This is because HAN-LCDs work only for the polarized light vibrating parallel to the LC direction. This study proposes a method for HAN-LCDs to work for non-polarized light.

Figure $2 \mathrm{~b}$ shows the alignment layers of high pretilt angles. Alignment layers with $\mathrm{a}$ high pretilt angle can be realized by a mixture of the polyimide material for homogeneous alignment layers and one for homeotropic alignment layers [8].

In Figure 3, the light passes through the single HAN-LCD from the incident angles $\Theta$ and $-\theta$, where $\Theta$ is the polar angle in the middle of the LCD. The p-wave of the incident light, from the direction $\Theta$, vibrates parallel to the long axis of the dichroic dye and is absorbed effectively.

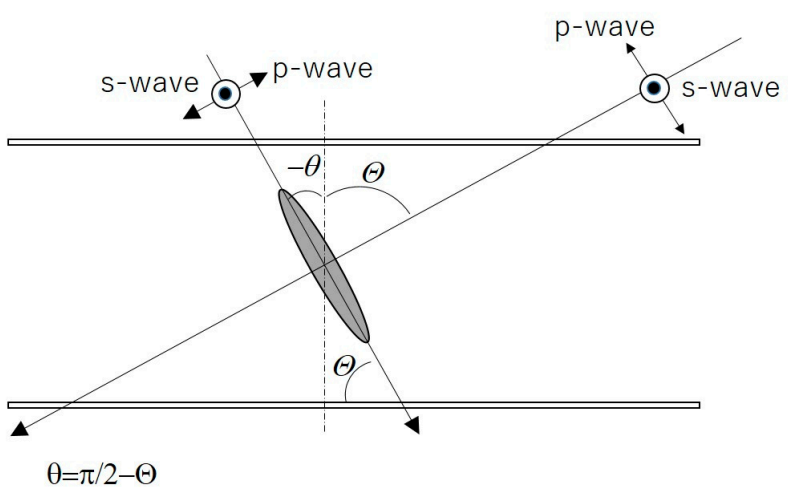

Figure 3. Light passing through the HAN or high pretilt angle LCDs from the incident angles of $\Theta$ and $-\theta$. 
In contrast, the vibration of the s-wave is perpendicular to the molecular axis and the absorption is small. For the incident light from the direction of $-\theta$, both the $\mathrm{p}$ - and s-waves vibrate perpendicularly to the molecular axis of the dye, resulting in the absorption being small. The transmittance, even from the $\Theta$ direction, is not sufficiently reduced owing to the presence of the s-wave.

The optical device shown in Figure 4 was proposed to realize a large ratio of the light strength from the $\Theta$ and $-\theta$ directions. By utilizing this device, it is possible to realize the ratio between the largest light strength from the $-\theta$ direction and the smallest one from the $\Theta$ direction.

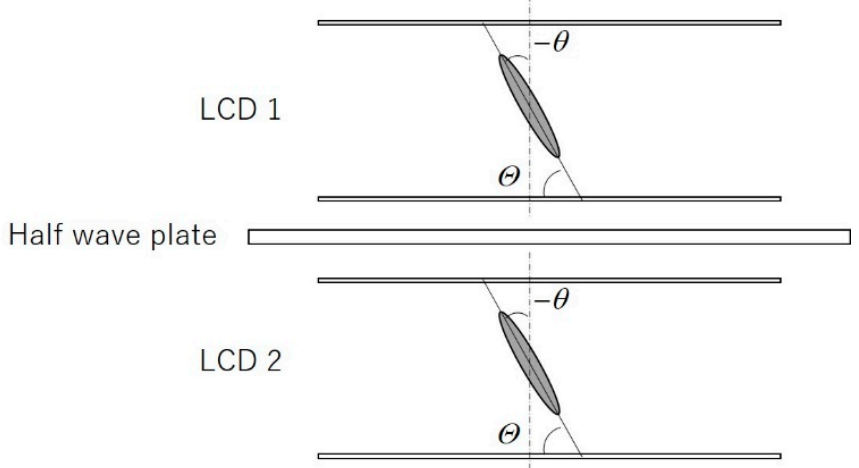

Figure 4. Structure of two-layer optically anisotropic LC filters.

Figure 5 shows the mechanism which causes the incident angle dependence of the transmittance. In the case of incident light to LCD1 from the $\Theta$ direction, the absorption of the p-wave is large, and the one of s-wave is small. By passing through the half-wave plate, the s-wave changes into a p-wave, which is effectively absorbed by passing through LCD2. However, in the case of incident light from the $-\theta$ direction, both $\mathrm{p}$ - and s-waves vibrate perpendicular to the dye molecular axis, passing through both LCD1 and LCD2 without large absorption. Therefore, the transmittance from the $\Theta$ direction becomes low and that from the $\theta$ direction becomes high. The device shown in Figure 4 would realize the largest ratio between the large light strength from the $\Theta$ direction and the smallest one from the $-\theta$ direction.

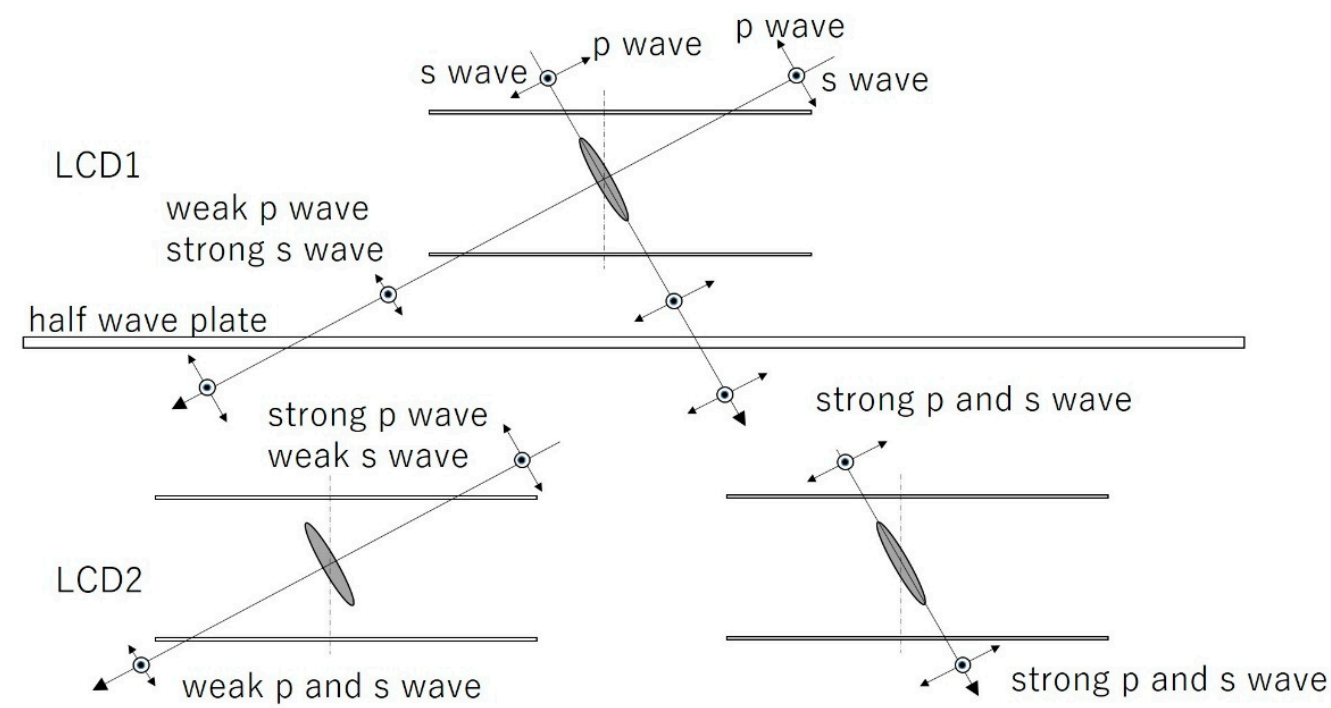

Figure 5. p-wave and s-wave through two-layer optically anisotropic LC filters in the direction of $\Theta$ and $-\theta$.

The demands for the devices which control transmittance depending on the incident angle exist in various situations. Especially, the demand to reduce only sunlight is usual. 
The function of the devices described in this paper should be desired for sunglasses, sunvisors, vehicle windows, and so on. Furthermore, the demand to show information to the limited direction also exists in the fields of information displays in vehicles, displays for games, and so on. The switching function is also effective for these purposes. The films named "louver film" also have a similar function. However, those films cannot show the switching function, and the incident angle for high transmittance is limited.

\section{Materials and Methods}

\subsection{HAN-LCD Preparations}

On the surfaces of two $2 \mathrm{~cm} \times 2 \mathrm{~cm}$ glass substrates and deposited $100 \Omega / \mathrm{cm}^{2}$ of ITO layer, alignment layers of $100 \mathrm{~nm}$ were formed. For one alignment layer, the polyimide of the homeotropic alignment layer, SE4811 (Nissan Chemical Co., Tokyo, Japan), was used. For another alignment layer, a mixture of two types of polyimides, PIA-X359-01X (85\%) of the homogeneous alignment layer and PIA-X768-01X (15\%) of the homeotropic alignment layer (JNC Petroleum Chem. Co. Tokyo, Japan), was used. The pretilt angle of the resultant polyimide layer was $6^{\circ}$.

The surfaces on both alignment layers were treated by an LC alignment process called "rubbing". Utilizing an adhesive containing silica spacers of $5 \mu \mathrm{m}$ radius, the two substrates were set, with the distance between the two alignment layers being $5 \mu \mathrm{m}$. The mixtures of LC material, ZLI-4792 (Merck Co. Darmstadt, Germany), and dichroic black dye NKX-4173 (Hayashibara Ltd., Okayama, Japan) or NKX-4010 (Hayashibara Ltd.) were injected at concentrations of 1,3 , and $5 \mathrm{wt} . \%$.

\subsection{High Pretilt Angle LCD Preparations \\ 2.2.1. $25^{\circ}$ Pretilt Angle}

Alignment layers with high pretilt angles were formed on the surfaces of the ITO layers by using a mixture of $80 \mathrm{wt} . \%$ polyimide PIA-X768-01X for the homeotropic alignment layer and $20 \mathrm{wt} . \%$ of polyimide PIA-X359-01X for the homogeneous alignment layers. Both alignment layers were treated by rubbing. The pretilt angle of the resultant alignment layer was $25^{\circ}$. The two substrates with the same alignment layers were set for opposite rubbing directions, as shown in Figure $2 b$.

\subsection{2. $40^{\circ}$ Pretilt Angle}

For the alignment layers, a mixture of 90\% PIA-X768-01X and 10\% PIA-X359-01X was used. The pretilt angle of the obtained alignment layer was $40^{\circ}$. LCDs were formed by using the same process.

\subsection{Preparations of Optically Anisotropic Optical Device Using Two-Layer LCDs}

For the half-wave plate, the film of Nichiban cellophane type No. 405, with a retardation of $260.4 \mathrm{~nm}(550 \mathrm{~nm})$, was attached to LCD1, as shown in Figure 4. The angle between the drawing direction of the film and the rubbing direction of the LCD1 alignment film was set to $45^{\circ}$. On the other surface of the film, LCD2 was set as shown in Figure 4 . The angle between the drawing direction of the film and the rubbing direction of LCD2 was set to $45^{\circ}$.

\subsection{Measurements of the Pretilt Angles for the Alignment Layers}

For the measurements of the alignment layer pretilt angles, LCD cells with parallel rubbing paths but opposite directions were prepared. The distance between the alignment layers was set as $20 \mu \mathrm{m}$. The pretilt angle was measured using the PAS-301 pretilt angle measurement system (Elscon Co., Newark, DE, USA).

\subsection{Measurements of the Incident Angle Dependence of the Transmittance}

The incident angle dependences of the transmittance of the LCDs and two-layer LCD devices were measured using an optical property measurement system RETS-100 
(Otsuka Electronics Co., Osaka, Japan), and the relationship between the sign of the incident angles and the directions of the polar angle of the LC molecules is shown in Figure 3. All measurements were carried out by using the light of $550 \mathrm{~nm}$ wavelength.

\section{Results and Discussions}

Transmittance Dependences on the Incident Angles for HAN-LCDs

Figure 6 shows the transmittance dependence on the incident angles for HAN-LCDs using LC materials containing 1,3, and $5 \mathrm{wt} . \%$ of the dichroic dye NKX-4173 (Hayashibara Ltd., Japan). In the case of $1 \mathrm{wt} . \%$, the maximum value, minimum value, and ratio between them was $71 \%, 55 \%$, and 1.3 respectively, in the range of $\pm 45^{\circ}$.

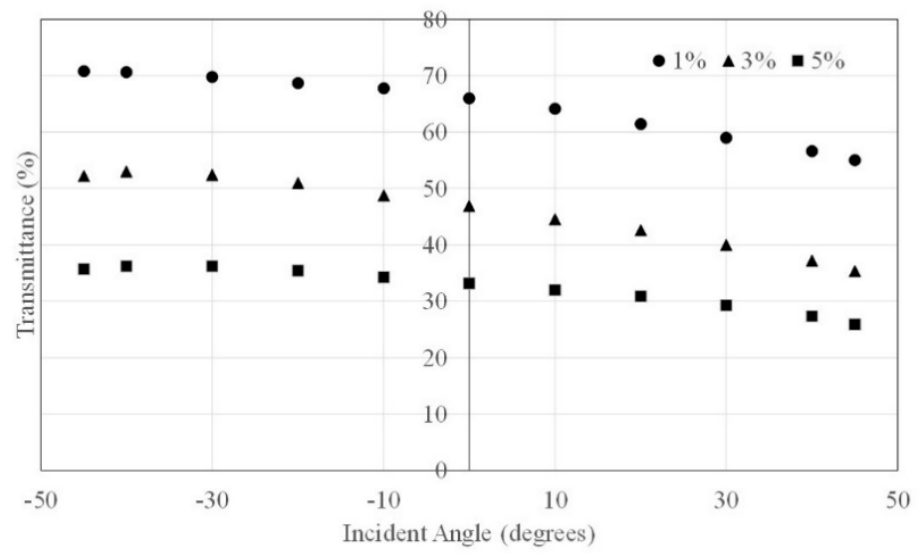

Figure 6. Transmittance dependences of the incident angles for HAN-LCDs using LC materials containing 1,3, and 5 wt.\% of NKX-4173 (Hayashibara Ltd., Japan) dichroic dye.

Figure 7 shows the dependence of the transmittance on the incident angles for the 2-layer HAN-LCDs, as shown in Figure 4, using LC materials containing 1, 3, and $5 \mathrm{wt} . \%$ of the dichroic dye NKX-4173 (Hayashibara Ltd., Japan). In the case of $1 \mathrm{wt} . \%$, the maximum value, minimum value, and ratio was $37 \%, 21 \%$, and 1.8 , respectively, in the range of $\pm 45^{\circ}$.

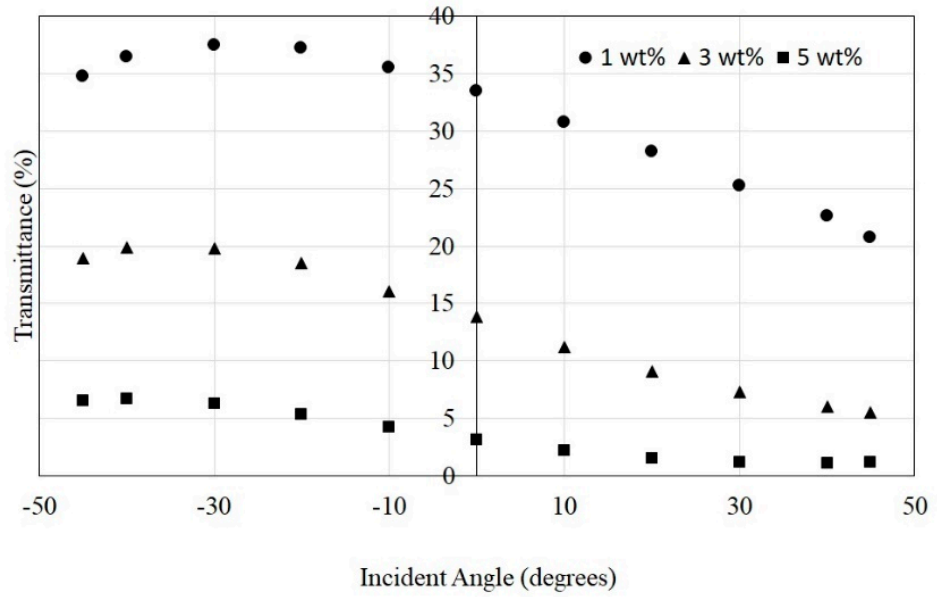

Figure 7. Transmittance dependences of the incident angles for the two-layer HAN-LCDs, as shown in Figure 4, using LC materials containing 1, 3, and 5 wt.\% of dichroic dye, NKX-4173 (Hayashibara Ltd., Japan).

Figure 8 shows the behaviors of the $\mathrm{p}$ - and s-waves parallel and perpendicular to the dye molecules for single and two-layer HAN-LCDs. In the case of HAN-LCDs, the polar angle of the LC molecules or dye molecules is distributed from several degrees in the vicinity of one alignment layer to $90^{\circ}$ in another. The polar angle of the LC molecule can be considered to represent either the angle in the middle of the LC layer or the average of the LC alignment. 

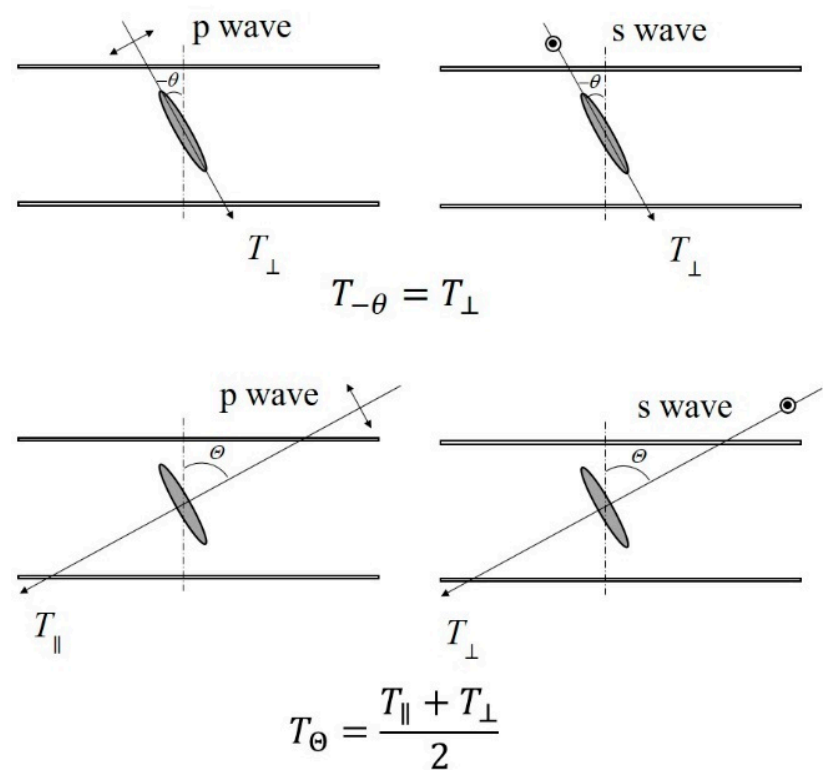

(a)
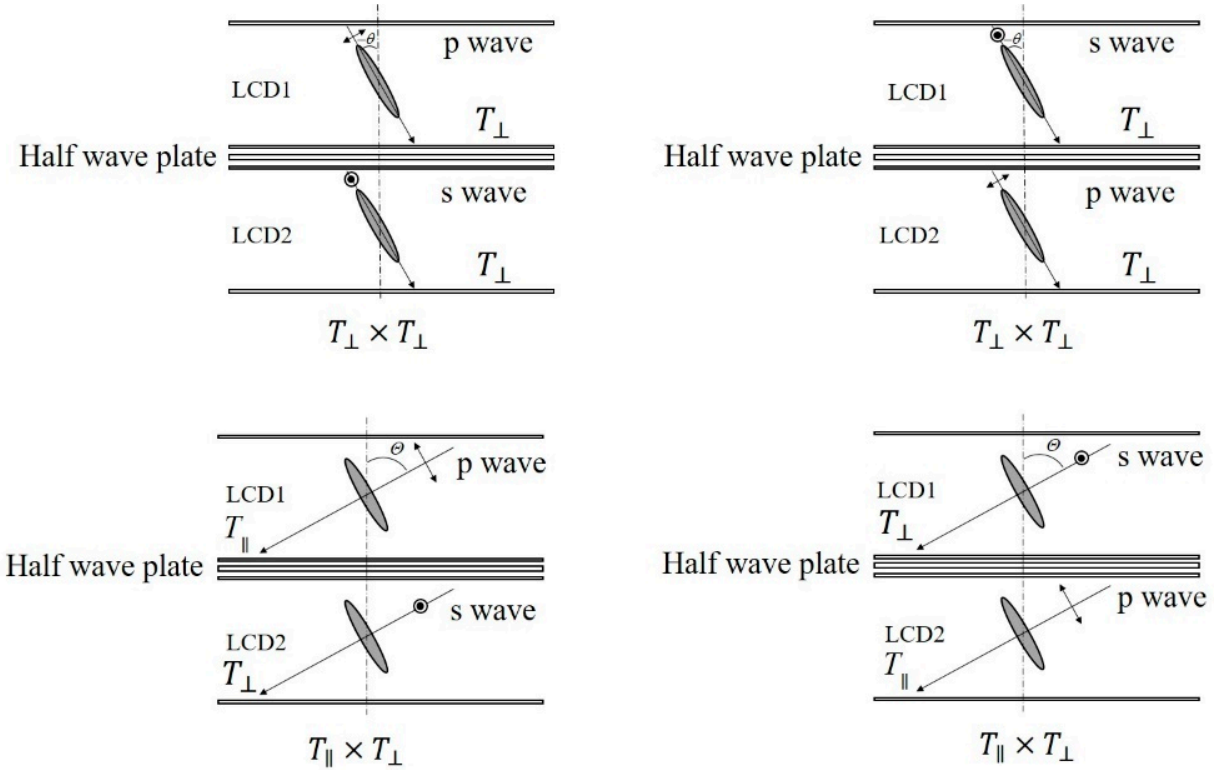

(b)

Figure 8. (a). Behaviors of p-waves and s-waves parallel and perpendicular to the dye molecules for single HAN-LCDs. (b). Behaviors of $\mathrm{p}$-waves and s-waves parallel and perpendicular to the dye molecules for 2-layer HAN-LCDs.

$T_{\|}$shows the transmittance of the polarized light vibrating parallel to the molecular axis of the dichroic dye through GH-LC materials. $T_{\perp}$ shows the transmittance of the polarized light vibrating perpendicular to the molecular axis of the dye.

$T_{\|}$and $T_{\perp}$ are the transmittances of the p-and s-waves from the angle $\Theta$ in Figure 8a, respectively. $T_{\perp}$ is almost equal to the transmittance $\mathrm{p}$-wave and s-wave from the angle $-\theta$. $T_{\Theta}$ and $T_{-\theta}$ are the transmittance of light from the incident angles $\Theta$ and $-\theta$, respectively. As shown in Figure 8a, for a single cell $T_{-\theta}$ is equal to $T_{\perp}$. In the case of light from the incident angle $\Theta$, the transmittance of the p-wave is equal to $T_{\|}$and that of the s-wave is equal to $T_{\perp}$. Generally, the strengths of the p- and s-waves are identical. Consequently, $T_{\Theta}$ can be represented by Equation (1):

$$
\left(T_{\|}+T_{\perp}\right) / 2
$$


and the ratio $r$, where $T_{\|}<T_{\perp}$, can be expressed as follows:

$$
r=\frac{T_{-\theta}}{T_{\Theta}}=\frac{2 T_{\perp}}{T_{\|}+T_{\perp}}
$$

Therefore, the ratio $r$ should be limited.

Figure $8 \mathrm{~b}$ shows the behavior of the $\mathrm{p}$ - and s-waves that enter into the two-layer HAN-LCDs from the incident angle $-\theta$ and $\Theta$. The transmittance of the p-wave through LCD1 from the incident angle $-\theta$ can be considered to be $T_{\perp}$, as described above. The $\mathrm{p}$-wave changes into the s-wave by passing through the half-wave plate. The transmittance of the s-wave is also considered to be equal to $T_{\perp}$. Therefore, the transmittance of the p-wave passing through two-layer LCDs is $T_{\perp}{ }^{2}$. The transmittance of the s-wave incident from $-\theta$ passing through two-layer HAN-LCDs is also equal to $T_{\perp}{ }^{2}$. As a result, the transmittance of the incident light from $-\theta$ should be $T_{\perp}{ }^{2}$.

The transmittance of the p-wave through LCD1 from the incident angle $\Theta$ can be expressed as $T_{\|}$. The $\mathrm{p}$-wave changes to the s-wave by a half-wave plate. As a result, the p-wave transmittance from the incident angle $\Theta$ can be represented by Equation (3):

$$
T_{\|} \times T_{\perp}
$$

In contrast, the s-wave transmittance for LCD1 is $T_{\perp}$ and changes to a p-wave by a half-wave plate. The p-wave transmittance through LCD2 is represented by Equation (3). Consequently, the transmittance of the incident light from $\Theta$ can be expressed by Equation (3).

The ratio $r$ of the maximum transmittance $T_{-\theta}$ and minimum transmittance $T_{\Theta}$ can be expressed as follows:

$$
r=\frac{T_{-\theta}}{T_{\Theta}}=\frac{T_{\perp}}{T_{\|}}
$$

For an increasing $T_{\perp}$ and $T_{\|}$ratio, it is effective to increase the dichroic ratio of the dye and the order parameters of the LC materials. Increasing the dye concentration and the cell thickness is effective in increasing the $r$ value by reducing the minimum transmittance, $T_{\Theta}$. The required maximum transmittance is determined depending on the application. Therefore, to achieve a large $r$ value, the dichroic ratio of the dye and the order parameters of the LC materials are important.

Figure 9 shows the dependence of the transmittance on the incident angles for HANLCDs using LC materials containing 1, 3, and $5 \mathrm{wt} . \%$ of the dichroic dye NKX-4173 (Hayashibara Ltd., Japan) with a polarizer. The optical axis of the polarizer was parallel to the alignment direction of the HAN-LCD. The transmittance was halved with the use of the polarizer. From Figure 9, it can be determined that $T_{\|}$was 0.32 , and $T_{\perp}$ was 0.62 for 1 wt.\%. For these values, the transmittance in Fig.9 was doubled because of the usage of the polarizer. By using these values for the two-layer LCD device, $T_{-\theta}=T_{\perp}^{2}$ becomes 0.38 and $T_{\Theta}=T_{\|} \times T_{\|}$becomes 0.20 . These values show the good correspondence with Figure 7 . By using these values, Figure 6 can also be approximately explained. In the case of 3 and $5 \mathrm{wt} . \%$, the relationship can also be approximately confirmed.

The absorbances $A_{\|}$and $A_{\perp}$ were measured by polarized light parallel and perpendicular to the alignment directions. Antiparallel LCD of $1^{\circ}$ pretilt angle using ZLI-4792 and NKX-4173 was used. $A_{\|}$and $A_{\perp}$ were 0.835 and 0.0725 , respectively. By Lambert-Beer's law, the ratio of the absorption coefficients of dichroic dye, $\alpha_{\|} / \alpha_{\perp}$, was $11.5\left(\alpha_{\|} / \alpha_{\perp}=A_{\|} / A_{\perp}\right)$. On the other hand, the transmittance of HAN LCD without a dichroic dye of $45^{\circ}$ incident angle was 0.785 . By using the value, the absorbances $A_{\|}$and $A_{\perp}$ of HAN GH-LCD of $45^{\circ}$ incident angle were calculated $\left(A_{\|}=0.39, A_{\perp}=0.102\right)$. The ratio of absorption coefficients of $45^{\circ}$ incident angle was 3.80 . The value was reduced to be $1 / 3$. This is considered to be the distribution of the dichroic dye polar angle from the homogeneous alignment layer to the homeotropic alignment layer. 


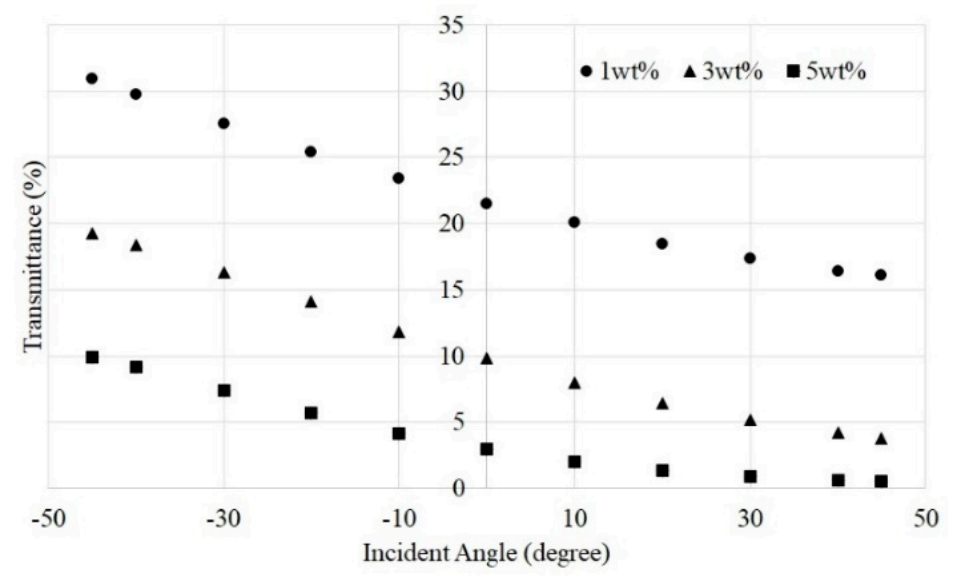

Figure 9. Transmittance dependences on the incident angles for HAN-LCDs using LC materials containing 1,3, and 5 wt.\% of NKX-4173 (Hayashibara Ltd. Japan) dichroic dye with a polarizer. The optical axis of a polarizer is parallel to the alignment direction of the HAN-LCD.

Figure 10 shows the transmittance dependencies on the incident angle for two-layer HAN-LCDs using LC materials containing $3 \mathrm{wt} . \%$ of dichroic dye with applied voltages of $0,1,3,5$, and $10 \mathrm{~V}$. The same voltage was applied to the two LC panels during the measurements. By applying a voltage, the polar angle of the LC molecules increased, resulting in the asymmetric properties of the LCDs decreasing. The saturated voltage of LCDs, using the LC material ZLI-4792, was approximately 4 V. However, the asymmetric properties were still observed at applied voltages as high as $10 \mathrm{~V}$ because of the effect of the pretilt angle and the LC molecular arrangement maintained in the vicinity of homogeneous alignment layer.

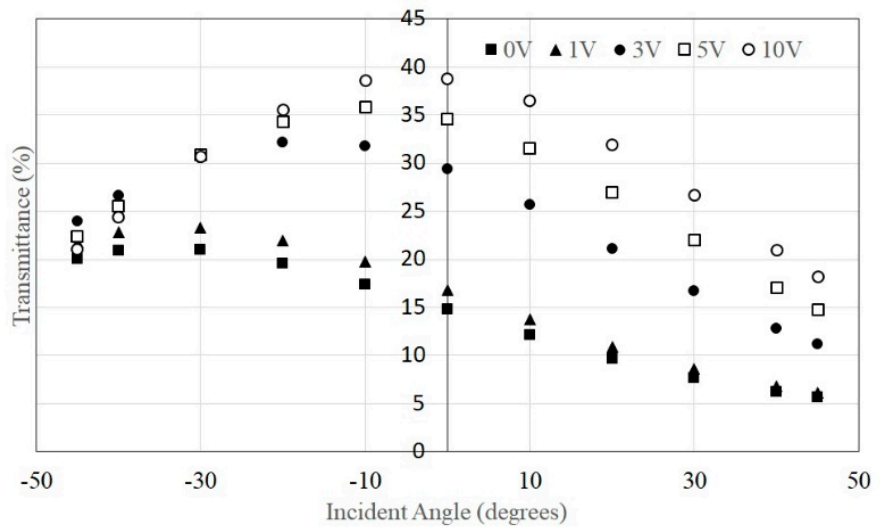

Figure 10. Incident angle dependencies of the transmittance for the two-layer HAN-LCDs using LC materials containing $3 \mathrm{wt} . \%$ of dichroic dye with applied voltages.

Figures 11 and 12 show the dependence of the transmittance on the incident angle for the two-layer LCDs, as shown in Figure 2b, with pretilt angles of $25^{\circ}$ and $40^{\circ}$, respectively. In the case of the two-layer LCDs with a $25^{\circ}$ pretilt angle, the maximum value was $28 \%$ at an incident angle of $-30^{\circ}$. The value at $45^{\circ}$ was $8 \%$ and the ratio $r$ was found to be 3.5 . In the case of two-layer LCDs with a $40^{\circ}$ pretilt angle, the maximum value was $35 \%$ at $-30^{\circ}$. The value at $45^{\circ}$ was $8 \%$ and the ratio $r$ was found to be 4.4 . In the case of two-layer HAN-LCDs with $1 \mathrm{wt} . \%$ dye concentration, the maximum value, the value of 45 degrees and the ratio were $37 \%, 21 \%$ and 1.8 , respectively. In the case of high pretilt angle LCDs, all the LC molecules were expected to be aligned in the same direction, although those of HAN-LCDs were distributed. As a result, the ratio of two-layer high pretilt angle LCDs was larger than that for HAN-LCDs. 


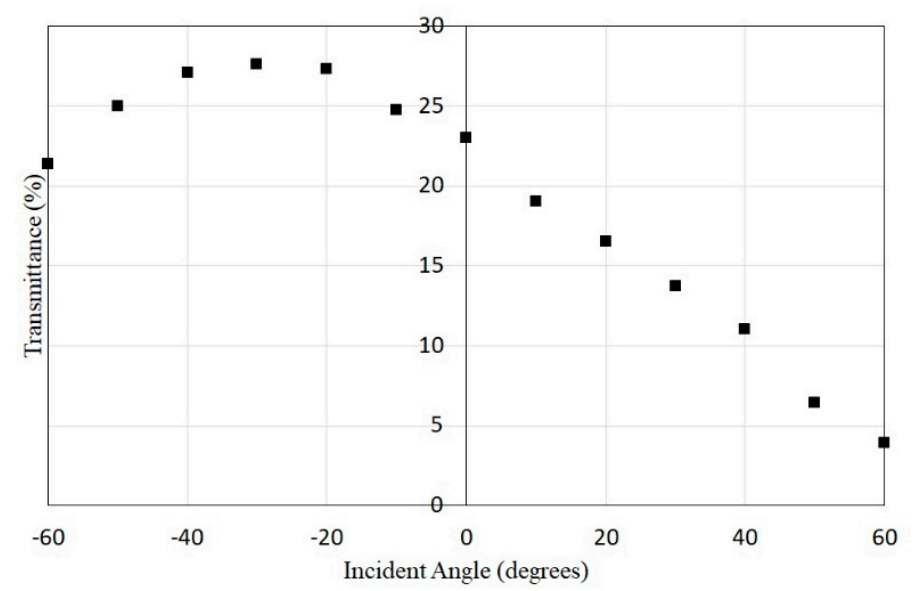

Figure 11. Transmittance dependence on the incident angle for the 2-layer LCD with a $25^{\circ}$ pretilt angle using a concentration of $1 \mathrm{wt} . \%$ NKX-4010 (Hayashibara Ltd., Japan).

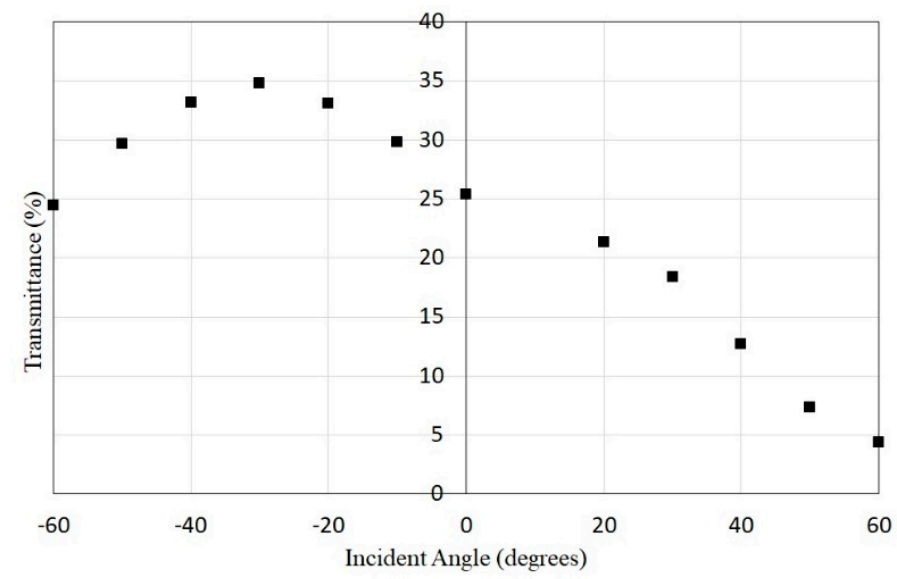

Figure 12. Transmittance dependence on the incident angle for the two-layer LCDs of $40^{\circ}$ pretilt angle. The concentration of NKX-4010 (Hayashibara Ltd., Japan) dye was 1 wt.\%.

In the case of high pretilt angle LCDs, the incident angle of the maximum value was approximately $30^{\circ}$. This is different from the incident angle, which was expected from the polar angle or pretilt angle. The incident angle of the maximum transmittance depends not only on the polar angle but also on the optical path length and reflection, as expected from the Fresnel law. Based on these three factors, both high pretilt two-layer LCDs were considered to show the maximum value at approximately $30^{\circ}$.

By using Fresnel's equations, the transmittance through glass (refractive index: 1.5) from air was calculated. When the transmittance perpendicular to the glass is 80 degrees, the transmittance for $\mathrm{p}$-wave/s-wave are $77 \% / / 76 \%$ at 30 degrees and $64 \% / 58 \%$ at 60 degrees, respectively. By increasing the incident angle, the transmittance decreases and the difference of the transmittance between p-wave and s-wave increases. In Figure 9, only the $p$-wave was used by a polarizer. By increasing the incident angle at $(-)$ direction, the transmittance increases monotonically. However, in Figures 7, 11 and 12, both s-waves and p-waves were used. This should be one of the reasons why the maximum value is observed. The same tendency is also observed in Figure 6.

\section{Conclusions}

This study proposed a series of devices capable of different transmittances based on incident angle direction. The devices consist of either two HAN GH-LCDs or GH-LCDs with a high pretilt angle antiparallel alignment and a half-wave plate between them. Both the $\mathrm{p}$ - and s-waves for the incident light can be controlled, owing to the rotation of the 
wave vibration direction by the half-wave plate. The dependences can be controlled by the applied voltage. The ratio of the devices using GH-LCDs with high pretilt antiparallel alignment was found to be higher than that using HAN GH-LCDs. This is because in the case of HAN GH-LCDs, the polar angle at the LC materials or dichroic dye is distributed from 2 or 3 degrees to 90 degrees.

Due to the capabilities of these devices, they have a wide range of application, including buildings, vehicles, and glasses.

Author Contributions: K.T. proposed and conceptualized the devices, supervised the project, and wrote the manuscript. S.S. and Y.T. prepared the sample cells and made measurements. M.I. supervised S.S. and Y.T. and analyzed the data. All authors have read and agreed to the published version of the manuscript.

Funding: This research received no external funding.

Institutional Review Board Statement: Not applicable.

Informed Consent Statement: Not applicable.

Data Availability Statement: Not applicable.

Acknowledgments: The authors would like to extend their sincerest gratitude to Chisso Petro Chemical Co. and Nissan Chemical Co. for providing the polyimide materials used during this investigation. The authors would also like to thank Merck Co. for providing the LC materials and Hayashibara Co. for the dye materials used during this investigation.

Conflicts of Interest: The authors declare no conflict of interest.

\section{References}

1. Yamaguchi, R.; Waki, Y.; Sato, S. Wide viewing angle properties in nematic liquid crystal/UV curable liquid crystal composite film with some aligned-modes. J. Photopol. Sci. Technol. 1997, 10, 19-24. [CrossRef]

2. Yamaguchi, R.; Inoue, K.; Oikawa, Y.; Takasu, T. Electro-optical property in hybrid aligned reverse mode cell using liquid crystals with positive and negative dielectric constant anisotropies. J. Photopol. Sci. Technol. 2017, 30, 463-466. [CrossRef]

3. Yamaguchi, R.; Ushizaki, R. Louver function in hybrid aligned reverse mode using dual frequency liquid crystal. J. Photopol. Sci. Technol. 2019, 32, 545-548. [CrossRef]

4. Hong, S.H.; Jeong, Y.H.; Kim, H.Y.; Lee, S.H. Novel nematic liquid crystal device associated with alignment controlled by fringe field. Jpn. J. Appl. Phys. 2001, 40, L272. [CrossRef]

5. Kasajima, Y.; Kato, T.; Kubono, A.; Tasaka, S.; Akiyama, R. Wide viewing angle of rubbing-free hybrid twisted nematic liquid crystal displays. Jpn. J. Appl. Phys. 2008, 47, 7941-7942. [CrossRef]

6. Lim, Y.J.; Song, J.H.; Kim, Y.B.; Lee, S.H. Single gap transflective liquid crystal display with dual orientation of liquid crystal. Jpn. J. Appl. Phys. 2004, 43, L972. [CrossRef]

7. Atorf, B.; Mühlenbernd, H.; Zentgraf, T.; Kitzerow, H. All-optical switching of a dye-doped liquid crystal plasmonic metasurface. Opt. Express 2020, 28, 8898-8908. [CrossRef] [PubMed]

8. Takatoh, K.; Akimoto, M.; Kaneko, H.; Kawashima, K.; Kobayashi, S. Molecular arrangement for twisted nematic liquid crystal displays having liquid crystalline materials with opposite chiral structures (reverse twisted nematic liquid crystal displays). $J$. Appl. Phys. 2009, 106, 64514. [CrossRef] 